\title{
Perceptual learning of cartoon faces by young children
}

\author{
FRANK S. MURRAY and REBECCA L. STANLEY \\ Randolph-Macon Woman's College, Lynchburg, Virginia 24503
}

\begin{abstract}
Children's perceptual learning of cartoon faces varying in facial features and orientations were investigated. After familiarization with the concept of "same-different," 54 children aged 4 to 6 years were given a pretest series of faces in a delayed matching task. Children in the experimental groups were then given attention training on one or more feature sets (eyes, noses, mouths), after which all children were given a posttest series. Results showed that training and age of subjects strongly affected children's matching responses in both pretest and posttest. Children given attention training on all features showed the greatest improvement in performance. Additionally, some transformations of forms were more difficult to match than others. Results were interpreted mainly to support cognitive-perceptual formulations of Bryant (1974) and Gibson (1969).
\end{abstract}

An important area of investigation in the perception of children concerns their ability to distinguish between forms of objects. Form perception may be seen as being influenced by factors such as internal features and orientations of features. The first factor, internal features, involves the distinction and abstraction of relevant features from the stimulus, which affect discrimination and memory for objects (Gibson, 1969). However, not all features are equally discriminable. A greater level of difficulty occurs in discrimination tasks involving comparisons of oblique lines with each other than in tasks in which obliques are compared with horizontal or vertical lines; this difficulty in discrimination of orientation of lines appears to diminish with increasing age of the child (Bryant, 1969, 1973; Rudel \& Teuber, 1963). The difficulty experienced by children with the perception of orientation of features may be related to the low degree of emphasis given to the importance of orientation in these situations. Experimental evidence has been reported showing that children can distinguish objects on the basis of orientation when instructed to choose the figure that appears "exactly the same" as a standard (Brooks \& Goldstein, 1963; Hendrickson \& Muehl, 1962; Murray \& Lee, 1977; Schaller \& Harris, 1974).

Previous research using young children has usually

This article was presented at the Tenth Annual Meeting of The Classification Society (North American Branch), April 10, 1979, at Gainesville, Florida. The authors would like to express their appreciation of the assistance and cooperation provided to them by the teachers of Villa Maria Academy, First Presbyterian Weekday School of Lynchburg, Virginia, and the Westminster Child Care Center of Charlottesville, Virginia. Requests for reprints should be sent to Frank S. Murray, Psychology Department, Randolph-Macon Woman's College, Lynchburg, Virginia 24503. focused on just one of these factors, without consideration of their interactions. Additionally, the stimuli used in these experiments have consisted of simple forms, that is, lines, geometric shapes, and schematic faces, which present a limited amount of content for perception (Brooks \& Goldstein, 1963; Bryant, 1969, 1973, 1974; Hendrickson \& Muehl, 1962; Murray \& McGuinn, 1977; Over \& Over, 1967; Rock, 1974; Rudel \& Teuber, 1963).

One mode of pictorial representation that offers distinct recognition of form and feature is the cartoon. According to Gibson (1969), cartoons emphasize distinctive features and reduce nonessential detail and may be perceived more readily than a photograph or a detailed line drawing. Experimental evidence from studies by Fraisse and Elkin (1963) and Ryan and Schwartz (1956) support this hypothesis. Research in our laboratory (Murray \& Lee, 1977; Murray \& McGuinn, 1977) has shown that children perform discrimination and matching tasks quite readily with schematic faces and that these faces are intrinsically interesting to them. It was the purpose of the present experiment to examine the effects of facial features and their orientations in cartoon faces on the perceptual learning of very young children.

\section{METHOD}

\section{Subjects}

A total of 63 children, 31 males and 32 females, participated in this experiment. Seven children were from the kindergarten of the Westminster Presbyterian Church of Charlottesville, Virginia; 38 children were from the kindergarten and first-grade classes of Villa Maria Academy of Lynchburg, Virginia; 18 were from the kindergarten of the First Presbyterian Weekday School of Lynchburg. Nine children were excluded from data analysis, five because of failure to meet criterion as stated below and four because of failure to follow instructions throughout the experiment. 
A total of 54 children, 26 males and 28 females, completed the experiment. There were three age groups with equal numbers of subjects (18) in each group. The mean age of the 4-year-olds was 4 years 7 months; the 5-year-old group had a mean age of 5 years 6 months; the 6 -year-old group had a mean age of 6 years 6 months.

\section{Materials and Apparatus}

Familiarization. For the familiarization task, stimuli were projected against a black background. The shapes, circles, squares, rectangles, and triangles, were painted red, yellow, violet, and green. Three of the slides required a simultaneous matching procedure; these slides showed a standard shape centered above four shapes in a horizontal array. Seven of the slides required a successive matching procedure: a geometric shape centered in the slide. When projected, these slides illuminated an area measuring $26.6 \times 38.1 \mathrm{~cm}$.

Pretest and posttest. Stimuli used for these tasks were projected images of 35-mm slides of cartoon faces drawn with black India ink on $8 \times 11$ in. $(20.32 \times 27.94 \mathrm{~cm})$ white tracing paper. The faces were elliptical, with major axis $17.5 \mathrm{~cm}$ and minor axis $12.0 \mathrm{~cm}$. Each face consisted of a combination of eyes, nose, and mouth; as there were three different types each of eyes, noses, and mouths, a total of 27 different faces were constructed. The eyes were ellipses, measuring $3 \mathrm{~cm}$ on the major axis and $1.5 \mathrm{~cm}$ on the minor axis. Embedded within each eye was a "pupil" made of a black polygon. Eye 1 was horizontal or parallel to the major axis of the facial ellipse. Eye 2 was oblique, with the left eye at a 145-deg angle and the right eye at a 35-deg angle to the minor axis, and Eye 3 was oblique, with the left eye at a 45-deg angle and the right eye at a 135-deg angle to the minor axis. The three noses were geometric shapes: Nose 1 was a circle with diameter of $3.5 \mathrm{~cm}$, Nose 2 was a diamond with sides of $2.5 \mathrm{~cm}$, and Nose 3 was a square with sides of $2.7 \mathrm{~cm}$. Each of these was attached to a "bridge" consisting of two parallel lines measuring $3 \mathrm{~cm}$. Mouth 1 was an ellipse with major axis $4.8 \mathrm{~cm}$ and minor axis $2 \mathrm{~cm}$. Mouth 2 was a semicircular shape $6.5 \mathrm{~cm}$ in length, $1.8 \mathrm{~cm}$ in width, with an arc of $115 \mathrm{deg}$; Mouth 3 was a 180-deg inversion of Mouth 2. The top of each face was painted black to represent hair. Figure 1 shows examples of the faces with the different features.

The faces were photographed against a white background under four 75-W bulbs with a Honeywell Pentax camera, using Kodachrome II film. One face was photographed per slide. Slides shown in the familiarization, pretest, and posttest stages were projected from a Kodak Carousel slide projector (Model 850 ) onto a $20 \times 20$ in. $(50.8 \times 50.8 \mathrm{~cm})$ white poster board. The projector was placed $6 \mathrm{ft}(1.83 \mathrm{~m})$ from the screen, so that the projected image of a face was $30.5 \mathrm{~cm}$ on the major axis and $20.32 \mathrm{~cm}$ on the minor axis against an illuminated background measuring $38.1 \times 26.6 \mathrm{~cm}$. The projector used a 300-W bulb.

Thirty-six pairs of slides were used in both the pretest and posttest. Half the slides showed "same" pairs of faces, and the
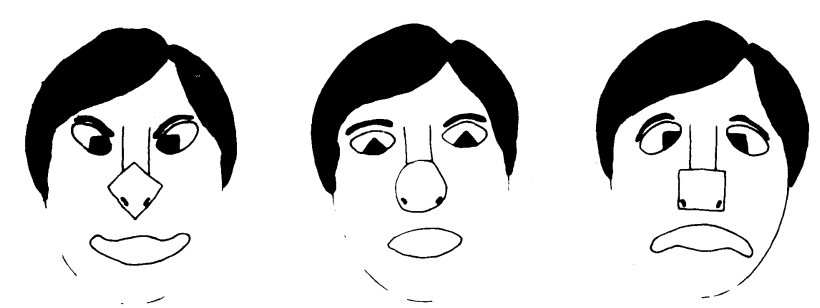

Figure 1. Stimulus faces showing examples of the three eyes, noses, and mouths used in pretest, training, and posttest. other 18 pairs showed "different" pairs of faces. Of the different pairs of slides, six pairs varied in one facial feature (e.g., eye, nose, or mouth), six pairs varied in two features (e.g., eye and nose, eye and mouth, or nose and mouth), and six pairs varied in three features. Slides were chosen so that each type of feature appeared an equal number of times within a series.

Training. The training task used a $25 \times 17.6 \mathrm{~cm}$ white wooden board on which was painted a black ellipse with major axis $20 \mathrm{~cm}$ and minor axis $16.5 \mathrm{~cm}$. Features similar to those of the cartoon faces were cut out of Masonite and were painted to match the original features; the Masonite pieces were the same sizes as the originals. Nine drawings of the original faces were mounted on white poster board; each member of the three sets of features appeared the same number of times in these drawings.

\section{Procedure}

Children were tested individually in a quiet room separate from their classrooms. They volunteered to participate in the experiment with permission of the teacher, who asked them if they wished to go with the experimenter (R.S.) to play "picture" games."

All subjects were given the same familiarization and pretest tasks. The familiarization was given to make sure the child understood the concept of "same-different." The experimenter read the following instructions: "Today I want you to play some picture games with me. I will show you some pictures on the wall and you will tell me some things about the pictures. If you do really well in the game, then you will win a little prize." At first, the experimenter showed the simultaneous matching slides. The child was instructed to look at the top shape and then to touch the bottom shape that looked just like it. If the child chose the correct figure, the experimenter said, "Good." If an incorrect response was given, the experimenter said, "That shape may look a little bit like the top shape, but it is not exactly like the top shape. If two shapes are not just like each other, then they are different. If they are the same, they look just alike." The experimenter then explained how the two shapes were different and then asked the child to choose the shape that was exactly the same as the top shape.

Slides requiring successive matching were then shown to the subject. The experimenter read the following instructions: "Now I will show you some shapes by themselves. Look at them carefully, for I will quickly make another shape appear on the screen. Then you tell me if the second shape is the same as the first shape, or if it is different (not the same)." The slides were grouped into three "same" and three "different" pairs; before each pair was shown, the experimenter held a board in front of the lens of the projector, so that no light was projected on the screen. The first of each slide pair was shown for $5 \mathrm{sec}$. A criterion of six out of six correct responses to the successive matching slides was set for all children to continue in the experiment.

Pretest. The 36 pairs of cartoon faces were shown to all children with these instructions: "Now we will play the same game but we will use different pictures. I will show you some cartoon faces; look at the first one, then I will show you the next face. Tell me if that face is the same or different from the first." As before, the experimenter covered the lens between slide pairs and exposed the first slide for $5 \mathrm{sec}$.

Training. After the pretest, children were randomly assigned to six groups of nine subjects each, with three members of each group in the six groups. The four experimental groups were trained with manipulation of eyes $\left(E_{e}\right)$, noses $\left(E_{n}\right)$, mouths $\left(E_{m}\right)$, or all three features $\left(E_{\text {enm }}\right)$. Two control groups were used: One group $\left(C_{p}\right)$ observed the nine training pictures of faces without any direction of attention to differences in features, and the other control group $\left(C_{\mathbf{x}}\right)$ was given an irrelevant task 
(they were asked to draw pictures of "something which they liked").

The four experimental groups were seated at a table in front of the board with elliptical outline. The painted Masonite features that each group was to manipulate were placed in an upright orientation by the board, so that the three eyes, noses, mouths, or all features were on the table.

The experimenter held up the first face mounted on poster board and asked the subject to take the eyes, nose, mouth, and so on, that matched the features on the face and to place them in the ellipse in the same place that they were on the face. Thus three of the experimental groups manipulated one feature (eyes, noses, or mouths) alone, and the fourth experimental group manipulated all three features. Nine presentations of faces were given; each of the three members of the different feature sets appeared three times in the series. Corrections were given by the experimenter for wrong features selected and for incorrect placement of features in the wrong area of the ellipse (mouth at the top, for example). Differences between features of a set were pointed out (the direction in which the eyes pointed, the position of the diamond nose compared with the square nose, etc.), and the child was continually instructed to choose the features that looked exactly like the ones on the face and to place them in the same position that they occupied on the poster board face.

The passive observation group $\left(\mathrm{C}_{\mathrm{p}}\right)$ was told that they would look at some drawings like the pictures they had just been shown (in the pretest) and then was shown each of the poster board drawings for $60 \mathrm{sec}$. The other control group $\left(C_{x}\right)$ was given a piece of paper and black crayon and was asked to draw a picture of "anything which you like." The duration of the training sessions was the same for all groups.

Posttest. All subjects were seated in front of the projector and asked if they would like to play the picture game with cartoons again. The same procedure and slides in the pretest were used in the posttest.

After the posttest, each subject was told that he had played the picture game well and was given a prize of candies. The total experimental procedure took about $35 \mathrm{~min}$.

\section{RESULTS AND DISCUSSION}

Analysis of results showed that training and age were important factors in the children's ability to classify cartoon faces in successive matching tasks. The number of errors made by subjects in the pretest and posttest were used in data analysis.

To determine if the children differed in performance on the pretest, a factorial analysis of variance for conditions and ages was computed. This analysis showed no significant difference between children later assigned to different training conditions $[F(5,36)=1.30]$. Age was found to have a significant effect on performance $[F(2,36)=27.48, p<.01]$; mean numbers of errors made by the 4-, 5-, and 6-year-old groups were 12.2, 10.3 , and 6.4 , respectively. A Newman-Keuls test for differences between age groups showed that 6-year-olds made significantly $(p<.05)$ fewer errors than 4 - and 5-year-olds.

A similar statistical analysis was computed using results from posttest measures. A significant difference was obtained among the training conditions $[\mathrm{F}(5,36)=$ $3.56, \mathrm{p}<.01]$. It appears that the special attention training aided children's performance (see Figure 2). The mean number of errors in the posttest for each of

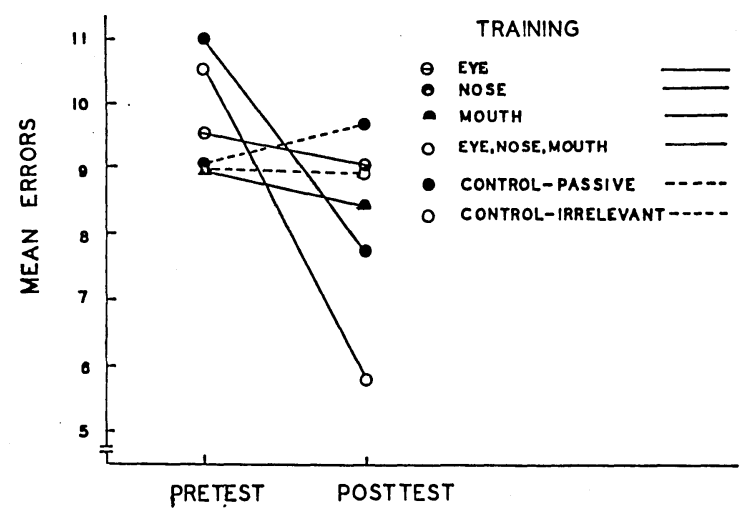

Figure 2. Mean errors for all groups on pretest and posttest.

the groups was as follows: Group $\mathrm{E}_{\mathrm{e}}$ (eye training), 9.1 errors; Group $\mathrm{E}_{\mathrm{n}}$ (nose), 7.9 errors; Group $\mathrm{E}_{\mathrm{m}}$ (mouth), 8.4 errors; Group $E_{\text {enm }}$ (all features), 5.8 errors; Group $C_{p}$ (passive observation), 9.7 errors; and Group $\mathrm{C}_{\mathrm{x}}$ (control), 8.8 errors. A Newman-Keuls test for differences between these groups revealed that Group $E_{e n m}$ was significantly different from all the other groups $(p<.05)$. Therefore, it appears that attention training on multifeatures was more effective in improving children's performances than were single features.

An analysis of covariance on the errors between pretest and posttest, using the pretest scores as covariates, for all conditions and ages showed that the posttest errors were significantly lower than pretest errors $[F(5,35)=4.67, p<.01]$. This improvement in matching occurred for all the experimental groups, but not for the two control groups. An analysis of the errors of the 18 cartoon faces that differed from each other in facial features revealed that the training was specific to the features the children were trained on. That is, if the child was trained to attend to eyes, he made very few errors on posttest to slides that differed on eyes and very little improvement in discriminating the other features. A significant difference between age groups for pretestposttest $[F(2,35)=6.47, p<.01]$ was also obtained. Individual comparison showed that the 5- and 6-yearolds made significantly fewer errors $(p<.05)$ than the 4-year-olds; no significant differences occurred between the 5- and 6-year-olds.

A comparison of errors between stimulus pairs differing in one, two, or three facial features was made, and, as expected, it was found that the number of features by which the faces varied produced significant differences in the number of errors in the pretest and posttest. For the pretest, a significant difference in errors to facial features $[F(2,36)=38.30, p<.01]$ was obtained; however, no significant difference was found among subjects in groups after they were randomly assigned to training conditions. A Newman-Keuls test indicated significant differences occurred when 
features differed by one and three variations and one and two variations. Significant differences among ages, conditions, and features in the posttest were also obtained. Apparently, children were able to use the feature training to distinguish between different stimuli in the posttest.

The effect of transformations of forms was investigated to see if certain transformations (rotations, obliques) were confused more often than were other transformations. To accomplish this, the number of errors was counted from stimuli that varied by one feature alone (i.e., eye, nose, or mouth) to see precisely what types of transformations (e.g., oblique to oblique vs. straight to oblique) were responsible for the most errors. With the eyes, the oblique-to-oblique transformation proved to be more difficult than the straightto-oblique transformation; the former had a mean error of $59 \%$ in the pretest and $56 \%$ in the posstest for all groups, whereas the straight-to-oblique transformation had a mean error of $41 \%$ in the pretest and $44 \%$ in the posttest. With the nose and mouth transformations, the mean percents of errors were about equal for both featural variations in the pretest and posttest. As shown in Figure 2 , the number of errors for posttest matches was less for all experimental groups, except for the group trained on the mouth feature; the numbers of errors were about equal for pretest and posttest. The highest mean percentage of errors occurred with the noses, and the lowest, with the mouths. This may have been due in part to the attention that children gave to different features. It may be noted that children frequently identified the faces as "happy" or "sad" according to the mouths; a total of 40 out of the 54 subjects labeled the faces in the pretest. Only two subjects commented that the noses were different shapes in the pretest. This use of a label to identify features is supported by experimental evidence that verbal labels aided discrimination tasks (Gibson, 1969; Murray \& Lee, 1977). It is likely that a child between 4 and 6 years of age would be familiar with the use of labels such as "happy" or "sad" for faces and be less familiar with geometric concepts such as a "diamond" (Bryant, 1974; Gibson, 1969).

The hypothesis that children do possess the perceptual capacity to distinguish different variations of features is evidenced by the significant difference among training conditions in the posttest and not in the pretest. Also, specific attention training seemed to be effective, as the group that received the most active training procedure, the manipulation of three features, had the lowest mean number of errors in the posttest. Therefore, children within this training condition were able to improve in their recognition of cartoon faces. Specific training on one feature did not appear to be as effective as training on several features, as the one-feature training groups did not differ significantly from each other or from the two control groups. However, the mean proportions of errors for all the experimental groups declined between pretest and posttest for each of the features, which indicates that initial difficulties in perception of the features and their transformations improved with attention training. In all statistical analyses in which age was tested as a factor, there was a significant difference among the errors made by the three age groups, with the greatest number being made by the youngest subjects, the 4-year-olds, and the lowest number being made by the 6-year-olds. This evidence relates to general hypotheses proposing a developmental trend of improved perceptual skills with increasing age. Bryant (1974) and Gibson (1969) suggest that this improvement is related to more efficient use of cognitive strategies by older children.

\section{REFERENCES}

Brooks, R. M., \& Goldstein, A. G. Recognition by children of inverted photographs of faces. Child Development, 1963, 34, 1033-1040.

Bryant, P. E. Perception and memory of the orientation of visually presented lines by children. Nature, 1969, 224, 1131-1132.

Bryant, P. E. What the young child has to learn about logic. In R. A. Hinde \& J. S. Hinde (Eds.), Constraints on learning. London: Academic Press, 1973.

Bryant, P. E. Perception and understanding in young children: An experimental approach. New York: Basic Books, 1974.

Fraisse, P., \& Elkin, E. H. Étude genetique de l'influence des modes de présentation sur le seuil de reconnaisance d'objets familiars. L'Année Physiologique, 1963, 63, 1-12.

Gibson, E. J. Principles of perceptual learning and development. New York: Appleton-Century-Crofts, 1969.

Hendrickson, L. N., \& Muehl, S. The effect of attention and motor response pretraining on learning to discriminate $b$ and $d$ in kindergarten children. Journal of Educational Psychology, $1962,53,236-241$.

Murray, F. S., \& Lee, T. S. The effects of attention-directing old children. Journal of Experimental Child Psychology, 1977, 23, 430-441.

Murray, F. S., \& McGuinn, P. K. Discrimination of features and orientations of schematic faces by children. Bulletin of the Psychonomic Society, 1977, 10, 283-286.

Over, R., \& Over, J. Detection and recognition of mirrorimage obliques by young children. Journal of Comparative and Physiological Psychology, 1967, 64, 467-470.

Rock, I. The perception of disoriented figures. Scientific American, 1974, 230, 78-85.

Rudel, R. G., \& Teuber, H. L. Discrimination of direction of line in children. Journal of Comparative and Physiological Psychology, 1963, 56, 892-898.

Ryan, T. A., \& Schwartz, C. B. Speed of perception as a function of mode of representation. American Journal of Psychology, 1956, 69, 60-69.

Schaller, M. J., \& Harris, L. Children judge "perspective" transformations of letterlike forms as different from prototypes. Journal of Experimental Child Psychology, 1974, 18, 226-241.

(Received for publication September 19, 1980.) 